\title{
Prognostic and predictive markers in glioblastoma and ALK overexpression
}

\author{
Jang-Hee Kim \\ Departments of Pathology, Ajou University School of Medicine, Suwon, Korea
}

Glioblastoma $(\mathrm{GBM})$ is the most common primary malignant brain tumor with a lethal clinical course [1]. Due to recent advances in medical knowledge and treatment modalities, survival of cancer patients has significantly improved. However, prognosis of patients with GBM remains dismal and less than $5 \%$ survive more than 5 years despite aggressive surgical resection and concurrent and adjuvant chemoradiation therapy [1-3]. This is the reason why new therapeutic approaches are urgently needed.

In this issue, Elsers et al. [4] reported anaplastic lymphoma kinase (ALK) and telomerase reverse transcriptase (TERT) expression in GBM and their clinical significance. The authors found ALK overexpression significantly correlated with $A L K$ gene alterations and TERT expression. In addition, ALK and TERT overexpression and $A L K$ gene alterations were associated with poor overall survival (OS) and progression-free survival (PFS), indicating that ALK overexpression could be an additional prognostic marker of GBM.

In GBM patients, age, performance status, extent of surgery, and histologic grade are generally considered prognostic factors [1]. With recent advances in the understanding of molecular pathogenesis of gliomas, certain molecular characteristics of gliomas have been included as prognostic markers [2,5]. Among those molecular alterations, isocitrate dehydrogenase $(I D H) \mathrm{mu}-$ tation status is considered as an important prognostic marker for GBM $[1,2,5]$. IDH mutation is typically identified in secondary GBM, which develops from a pre-existing glioma through malignant transformation. Patients with $I D H$-mutant GBM are younger and have a significantly longer survival than patients

Received: April 27, 2021 Accepted: April 29, 2021

Corresponding Author: Jang-Hee Kim, MD

Department of Pathology, Ajou University School of Medicine, 206 World cup-ro, Yeongtong-gu, Suwon 16499, Korea

Tel: +82-31-219-5925, Fax: +82-31-219-5934, E-mail: drjhk@ajou.ac.kr with $I D H$-wild type GBM $[1,2]$. IDH is an enzyme involved in the tricarboxylic acid cycle. IDH mutations alter enzymatic activity resulting in production of the oncometabolite, 2-hydroxyglutarate, which can cause tumor-driving epigenetic changes [6]. To date, a target agent for mutant IDH is not available. However, the development of therapies specific for $I D H$ mutations will lead to a fundamental change in the treatment of GBM [5].

The blood brain barrier (BBB) is a major obstacle in the development of new drugs for brain tumors. Most chemotherapeutic drugs cannot penetrate the BBB and only a limited number of drugs can be used in treatment of GBM [3,5]. Temozolomide, an alkylating agent that can penetrate the $\mathrm{BBB}$, is currently included in the standard GBM therapy $[1,3,5]$. Temozolomide induces alkylation or methylation of DNA frequently at the $\mathrm{N}^{7}$ - or $\mathrm{O}^{6}$-position of guanine residues, which causes cytotoxicity and death of tumor cells. However, tumor cells with $\mathrm{O}^{6}$-methylguanine-DNA methyltransferase (MGMT) can remove the DNA alkyl group induced by temozolomide, rendering tumor cells resistant to temozolomide [3]. Therefore, MGMT activity status in GBM is considered an important predictive marker of therapeutic effects caused by alkylating agents. Methylation in the promoter region of MGMT can abolish MGMT activity; therefore, analysis of the methylation status in the promoter region of MGMT is currently performed in GBM patients to predict the response to temozolomide [1-3,5].

ALK is a protein with tyrosine kinase activity and encoded by the $A L K$ gene located on chromosome 2 [7]. ALK gene alterations can promote carcinogenesis [8] and have been reported in various tumors, including anaplastic large cell lymphoma [9], melanoma [8], neuroblastoma [10], and a subset of non-small cell lung carcinoma [11]. The most frequent $A L K$-related genetic aberrations are translocations [12]. In recent studies, ALK overexpression in GBM reportedly ranged from $30 \%-70 \%[4,12,13]$. 
Chiba et al. [14] suggested a possible biological role of ALK in stimulating proliferation and neovascularization in GBM. Dalia et al. [4] showed that ALK overexpression in GBM was significantly associated with proliferation of tumor cells, poor OS, and PFS, indicating a prognostic role in GBM. However, data on the prognostic role of ALK in GBM are very limited and remain controversial $[4,12,13]$. To properly evaluate the prognostic role of ALK in GBM, further investigative studies with large cohorts are needed.

Chemotherapeutic agents targeting $A L K$ genetic alterations (e.g., EML4-ALK gene fusion) have been used in clinical practice for treatment of ALK-positive lung cancer and patients showed improved OS and PFS [15]. In GBM, pre-clinical and in vivo studies showed positive outcomes with application of ALK inhibitors in GBM [16-19]. To date, no clinical trials of ALK inhibitors for the treatment of GBM have shown a significant effect on the survival of GBM patients, probably due to low BBB penetration of ALK inhibitors and difficulties in achieving adequate therapeutic concentration in the brain [13,16-19]. However, development of new ALK inhibitors that can penetrate the BBB is ongoing, and if ALK overexpression can predict sensitivity to new ALK inhibitors, ALK overexpression will be an additional important predictive marker in the treatment of GBM.

\section{Ethics Statement}

Not applicable.

\section{Availability of Data and Material}

Data sharing not applicable to this article as no datasets were generated or analyzed during the study.

\section{Code Availability}

Not applicable.

\section{ORCID}

Jang-Hee Kim https://orcid.org/0000-0001-5825-1361

\section{Conflicts of Interest}

J.-H.K., a contributing editor of the Journal of Pathology and Translational Medicine, was not involved in the editorial evaluation or decision to publish this article.

\section{Funding Statement}

No funding to declare.

\section{References}

1. Louis DN, Ohgaki H, Wiestler OD, Cavenee WK. WHO classification of tumours of the central nervous system. Revised 4th ed. Lyon: IARC Press, 2016; 28-56.

2. Eckel-Passow JE, Lachance DH, Molinaro AM, et al. Glioma groups based on $1 \mathrm{p} / 19 \mathrm{q}, I D H$, and TERT promoter mutations in tumors. $\mathrm{N}$ Engl J Med 2015; 372: 2499-508.

3. Hegi ME, Diserens AC, Gorlia T, et al. MGMT gene silencing and benefit from temozolomide in glioblastoma. N Engl J Med 2005; 352: 997-1003.

4. Elsers D, Temerik DF, Attia AM, Hadia A, Hussien MT. Prognostic role of ALK-1 and h-TERT expression in glioblastoma multiforme: correlation with $A L K$ gene alterations. J Pathol Transl Med 2021; 55: 212-224.

5. Aldape K, Zadeh G, Mansouri S, Reifenberger G, von Deimling A. Glioblastoma: pathology, molecular mechanisms and markers. Acta Neuropathol 2015; 129: 829-48.

6. Gross S, Cairns RA, Minden MD, et al. Cancer-associated metabolite 2-hydroxyglutarate accumulates in acute myelogenous leukemia with isocitrate dehydrogenase 1 and 2 mutations. J Exp Med 2010; 207: 339-44.

7. Morris SW, Naeve C, Mathew P, et al. ALK, the chromosome 2 gene locus altered by the $t(2 ; 5)$ in non-Hodgkin's lymphoma, encodes a novel neural receptor tyrosine kinase that is highly related to leukocyte tyrosine kinase (LTK). Oncogene 1997; 14: 2175-88.

8. Wiesner T, Lee W, Obenauf AC, et al. Alternative transcription initiation leads to expression of a novel ALK isoform in cancer. Nature 2015; 526: 453-7.

9. Yu R, Chen G, Zhou C, et al. Extra copies of ALK gene locus is a recurrent genetic aberration and favorable prognostic factor in both ALK-positive and ALK-negative anaplastic large cell lymphomas. Leuk Res 2012; 36: 1141-6.

10. Janoueix-Lerosey I, Lopez-Delisle L, Delattre O, Rohrer H. The ALK receptor in sympathetic neuron development and neuroblastoma. Cell Tissue Res 2018; 372: 325-37.

11. Wu SG, Kuo YW, Chang YL, et al. EML4-ALK translocation predicts better outcome in lung adenocarcinoma patients with wild-type EGFR. J Thorac Oncol 2012; 7: 98-104.

12. Karagkounis G, Stranjalis G, Argyrakos T, et al. Anaplastic lymphoma kinase expression and gene alterations in glioblastoma: correlations with clinical outcome. J Clin Pathol 2017; 70: 593-9.

13. Franceschi E, De Biase D, Di Nunno V, et al. The clinical and prognostic role of ALK in glioblastoma. Pathol Res Pract 2021; 221: 153447.

14. Chiba R, Akiya M, Hashimura M, et al. ALK signaling cascade confers multiple advantages to glioblastoma cells through neovascularization and cell proliferation. PLoS One 2017; 12: e0183516.

15. Shaw AT, Bauer TM, de Marinis F, et al. First-line lorlatinib or crizotinib in advanced ALK-positive lung cancer. N Engl J Med 2020; 383: 2018-29.

16. Goodwin CR, Rath P, Oyinlade O, et al. Crizotinib and erlotinib inhibits growth of c-Met(+)/EGFRvIII(+) primary human glioblastoma xenografts. Clin Neurol Neurosurg 2018; 171: 26-33.

17. Greish K, Jasim A, Parayath N, et al. Micellar formulations of crizotinib and dasatinib in the management of glioblastoma multiforme. J Drug Target 2018; 26: 692-708.

18. Das A, Cheng RR, Hilbert ML, et al. Synergistic effects of crizotinib and temozolomide in experimental FIG-ROS1 fusion-positive glioblastoma. Cancer Growth Metastasis 2015; 8: 51-60.

19. Le Rhun E, Chamberlain MC, Zairi F, et al. Patterns of response to crizotinib in recurrent glioblastoma according to ALK and MET molecular profile in two patients. CNS Oncol 2015; 4: 381-6. 